ISSN 0103-9954

\title{
PRODUÇÃO DE PAINÉIS DE CIMENTO-MADEIRA COM ADIÇÃO DE DIFERENTES PROPORÇÕES DE CASCA E MICROSSÍLICA
}

\section{PRODUCTION OF WOOD-CEMENT BONDED PARTICLEBOARDS WITH DIFFERENT CONTENTS OF BARK AND MICROSILICA}

\author{
Gilmar Correia Silva ${ }^{1}$ João Vicente de Figueiredo Latorraca ${ }^{2}$ Roberto Carlos Costa Lelis ${ }^{2}$ \\ Divino Eterno Teixeira ${ }^{3}$ Jair Figueiredo do Carmo ${ }^{1}$ Érika da Silva Ferreira ${ }^{1}$
}

\section{RESUMO}

O objetivo deste trabalho foi avaliar o efeito da adição de microssílica em três porcentagens $(0,20 \mathrm{e}$ $30 \%$ ) sobre as propriedades físicas e mecânicas de painéis cimento-madeira de Eucalyptus urophylla compostos por três relações madeira:casca (100:0, 95:5 e 90:10). Os resultados mostraram que o efeito mais significativo do aditivo sobre os painéis produzidos ocorreu naqueles que continham casca, e que a adição de $20 \%$ do aditivo foi mais eficiente sobre as propriedades físicas e mecânicas.

Palavras-chave: microssílica; painéis de cimento-madeira; Eucalyptus urophylla.

\begin{abstract}
This work's objective was to evaluate the effect of three percentages of addition of microsilica $(0,20$ and $30 \%$ ) on the physical and mechanical properties of wood-cement bonded particleboards of Eucalyptus urophylla composed by three wood:bark ratios (100:0, 95:5 and 90:10). Results showed that the most significant effect of the additive on the produced panels was in those containing bark, and that of $20 \%$ of additive was more efficient on the physical and mechanical properties.
\end{abstract}

Key words: microsilica; wood-cement bonded particleboard; Eucalyptus urophylla.

\section{INTRODUÇÃO}

Em função de suas características, os painéis de cimento-madeira têm seu uso ampliado para além daqueles em que são aplicados os painéis convencionais, apresentando grandes possibilidades de uso, especialmente em ambientes úmidos e com riscos de incêndios (Chapola, 1989).

Os aditivos minerais, como a sílica ativa ou microssílica, são um dos mais promissores aditivos para uso em painéis minerais (Lange et al., 1989). A substituição do cimento Portland por várias porções de sílica possibilita melhorias nas propriedades de resistência de painéis produzidos partindo de espécies de baixa compatibilidade com o aglomerante. Entretanto, a efetiva proporção de sílica para substituir o cimento na mistura depende da aptidão da espécie.

A microssílica é um material extraído partindo da escória da fabricação de ligas metálicas, cinzas de cascas de arroz e de material restante das colheitas de grãos. O aditivo é caracterizado como um material extremamente fino, de 10 a 100 vezes menor que o grão de cimento, que preenche vazios entre os grãos maiores, propiciando uma estrutura mais compacta, reagindo com a cal livre melhorando a resistência e durabilidade do compósito.

A adição do aditivo deixa o compósito mais impermeável e, em contato com hidróxido de cálcio, liberado pela hidratação do cimento, resulta na formação do silicato de cálcio hidratado (C-S-H). Essa reação provoca uma redução considerável do tamanho dos poros. Ao mesmo tempo, eles ficam praticamente incomunicáveis entre si, impedindo a passagem de fluídos, melhorando significativamente a qualidade de cristalinização do cimento, tornando-o muito mais resistente à compressão e à tração (Latorraca, 2000).

Estudos realizados por Pardo et al. (2001) mostraram que a microssílica em contato com o cimento já

1. Acadêmico do Programa de Pós-graduação em Ciências Ambientais e Florestais, Instituto de Florestas, Universidade Federal Rural do Rio de Janeiro da UFRRJ, Caixa Postal 74527, CEP: 23890-000, Seropédica (RJ).

2. Engenheiro Florestal, Dr., Professor do Departamento de Produtos Florestais, Instituto de Florestas, Universidade Federal Rural do Rio de Janeiro da UFRRJ, Caixa Postal 74527, CEP: 23890-000, Seropédica (RJ).

3. Engenheiro Florestal, Analista Ambiental do Laboratório de Produtos Florestais, Instituto Brasileiro do Meio Ambiente (IBAMA), CEP: 70000-000, Brasília (DF).

Recebido para publicação em 3/11/2004 e aceito em 10/02/2006. 
hidratado, reage transformando a sílica amorfa em cristobalita, acompanhada pela retração da matriz do compósito. A retração da matriz, que não é compensada pelos agregados presentes (que formam uma estrutura rígida), pode resultar em poros na microestrutura. Essa microestrutura promove a presença de espaços disponíveis para o escoamento do fluido.

O objetivo deste trabalho foi avaliar o efeito da adição de aditivo mineral (microssílica) sobre as propriedades físicas e mecânicas de painéis cimento-madeira produzidos com três composições de madeira:casca.

\section{MATERIAL E MÉTODOS}

\section{Matéria-Prima}

Cinco árvores da espécie Eucalytptus urophylla foram coletadas em um povoamento da FLONA Mário Xavier - IBAMA, localizada no município de Seropédica, RJ. O aglutinante empregado na composição foi o cimento Portland ARI e como aditivo químico o Cloreto de cálcio $\left(\mathrm{CaCl}_{2}\right)$. Foi aplicado ainda o aditivo mineral microssílica.

\section{Parâmetros do Processo}

Foram utilizados os seguintes parâmetros no estudo das propriedades dos painéis:

- Relação madeira:cimento $=1: 2,75$

- Relação água:cimento $=1: 2,50$ ( $40 \%$ de água com base no peso do cimento)

- Aditivo químico $=4 \%$ (com base no peso do cimento)

- Aditivo mineral = porcentagens aplicadas com base no peso do cimento $(0,20$ e $30 \%)$

- Massa específica nominal do painel $=1,25 \mathrm{~g} / \mathrm{cm}^{3}$

- Dimensão do painel $=40 \mathrm{~cm}$ largura $\times 40 \mathrm{~cm}$ comprimento $\times 1,6 \mathrm{~cm}$ espessura

- Parâmetros de prensagem:

$$
\begin{aligned}
& \text { - pressão específica }=3,92 \mathrm{MPa} \\
& \text { - temperatura de prensagem }=\text { ambiente } \\
& \text { - tempo de grampeamento }=24 \mathrm{~h} \\
& \text { - acondicionamento }=27 \text { dias }
\end{aligned}
$$

\section{Processo de Produção dos Painéis}

Foram produzidos painéis partindo das relações madeira:casca: 100:00, 95:05 e 90:10. Para cada relação foram adicionadas as seguintes porcentagens de aditivo mineral: 0, 20 e $30 \%$.

As seguintes fases foram seguidas para manufatura dos painéis: determinação da densidade básica da madeira; cálculo dos compostos do painel; obtenção e preparação das partículas; formação do colchão e prensagem dos painéis e acondicionamento.

\section{Determinação da massa específica básica da madeira}

Por meio dos discos extraídos foram coletadas amostras para determinação da massa específica básica da madeira. Para tanto, foram retirados discos a 0, 25, 50, 75 e $100 \%$ da altura total do tronco e um disco extra no diâmetro à altura do peito (DAP). O método empregado na determinação do volume das amostras foi o da balança hidrostática.

\section{Cálculo dos compostos do painel}

A metodologia utilizada para se efetuar os cálculos dos compostos de cada painel foi a sugerida por Souza (1994). Os parâmetros necessários para o cálculo de um painel são: comprimento, largura e massa específica do painel ( $0 \%$ ), conteúdo de umidade da madeira (u.m.), relação de madeira:cimento, relação de água:cimento e taxa de água de hidratação-cimento.

\section{Obtenção e preparo das partículas de madeira e da casca}

As toras de 2,0 m foram descascadas e seccionadas em discos de 3,6 cm de espessura e reduzidos em blocos com as dimensões compatíveis com a capacidade do gerador de partículas. Posteriormente, as partículas de madeira e a casca foram moídas em moinho de martelo e secas ao ar livre. Para efeito do 
cálculo da quantidade de água de cada painel, foi necessário medir o teor de umidade das partículas, que foi realizado pelo método da pesagem.

Utilizando-se uma peneira mecânica, foi obtida a fração granulométrica cujas partículas passaram pela peneira de $2,08 \mathrm{~mm}$ de abertura e ficaram retidas na peneira de $0,61 \mathrm{~mm}$ de abertura.

\section{Formação do colchão e prensagem dos painéis}

Devidamente calculados e pesados, o cimento, as partículas de madeira e/ou casca, água e o aditivo químico foram misturados em uma betoneira de eixo inclinado. Inicialmente foram colocadas as partículas de madeira que foram lentamente pulverizadas com a água contendo o aditivo químico diluído. Em seguida, foi adicionado o cimento sobre as partículas até homogeneização da mistura.

A seguir, o material foi retirado da betoneira, sendo a massa de cada painel devidamente pesada, separada e distribuída aleatoriamente na caixa formadora do colchão com as dimensões de $40 \mathrm{~cm}$ de largura e $40 \mathrm{~cm}$ de comprimento. Essa caixa foi colocada sobre uma chapa de alumínio untada com óleo mineral de baixa viscosidade para facilitar a retirada do painel após sua prensagem e grampeamento. Retirando-se a caixa, duas barras de ferro de $16 \mathrm{~mm}$ foram colocadas sobre a chapa para o controle da espessura final. A seguir, foi sobreposta outra chapa de alumínio também untada com óleo. Já carregada com o painel e os aparatos para o grampeamento, a prensa foi fechada a uma pressão específica de 3,92 MPa. Após grampear os painéis, transcorreu-se a fase de restrição por grampeamento. Passadas 24 horas, os grampos foram retirados e os painéis identificados e encaminhados para fase de acondicionamento.

\section{Acondicionamento}

Os painéis foram acondicionados em câmara climática com temperatura e umidade relativa controladas $\left(20 \pm 1^{\circ} \mathrm{C}\right.$ e $\left.65 \pm 5 \%\right)$. Para todos os tratamentos, do início da prensagem até os ensaios dos corpos-de-prova, foram transcorridos 28 dias.

\section{Testes Mecânicos e Físicos dos Painéis}

As propriedades dos painéis foram avaliadas de acordo com a norma americana ASTM D - 1037 (1982) e a norma alemã DIN 52362 (1982), sendo adotado os seguintes ensaios:

Propriedades Mecânicas

- Flexão estática (FE) - Módulo de elasticidade (MOE) e Módulo de ruptura (MOR): teste de acordo com a norma DIN 52362

- Ligação interna (LI) - teste de acordo com a norma ASTM D1037

\section{Propriedades Físicas}

- Inchamento em Espessura (IE) - teste de acordo com a norma ASTM D1037

- Absorção de água (AA) - teste de acordo com a norma ASTM D1037

- Massa específica aparente dos Painéis - dimensões e massa dos corpos-de-prova

Para obtenção da massa específica aparente de cada painel, determinou-se, inicialmente, a massa específica aparente de cada corpo-de-prova, obtendo-se a seguir a média para cada tratamento.

\section{Delineamento Experimental e Análise Estatística}

$\mathrm{O}$ experimento seguiu o delineamento inteiramente ao acaso com arranjo fatorial do tipo $3 \times 3$ (relação madeira:casca $\mathrm{x}$ percentagem de aditivo), totalizando nove tratamentos e, com três repetições, um total de 27 painéis produzidos (Tabela 1).

Os resultados foram analisados estatisticamente por meio de análise de variância (ANOVA). O uso da análise de covariância (ANCOVA) foi condicionado ao resultado da ANOVA aplicada à massa específica dos painéis. O teste de Tukey a $95 \%$ de probabilidade foi empregado, todas as vezes que a hipótese de nulidade foi rejeitada. 
TABELA 1: Tratamentos empregados para a composição dos painéis cimento-madeira.

TABLE 1: Treatments of the wood-cement bonded particleboards.

\begin{tabular}{c|c|c|c}
\hline Tratamento & Relação madeira:casca & Aditivo mineral (\%) & Repetições \\
\hline $\mathrm{T}_{1}$ & \multirow{2}{*}{$100: 0$} & 0 & 3 \\
$\mathrm{~T}_{2}$ & & 20 & 3 \\
$\mathrm{~T}_{3}$ & \multirow{3}{*}{$95: 5$} & 30 & 3 \\
\hline $\mathrm{T}_{4}$ & & 0 & 3 \\
$\mathrm{~T}_{5}$ & & 20 & 3 \\
$\mathrm{~T}_{6}$ & \multirow{3}{*}{$90: 10$} & 0 & 3 \\
\hline $\mathrm{T}_{7}$ & & 20 & 3 \\
$\mathrm{~T}_{8}$ & 30 & 3 \\
$\mathrm{~T}_{9}$ & & 0 & 3 \\
\hline
\end{tabular}

\section{RESULTADOS E DISCUSSÃO}

\section{Massa Específica Básica da Madeira}

A massa específica básica obtida para a madeira de Eucalyptus urophylla foi de $0,44 \mathrm{~g} / \mathrm{cm}^{3}$ aos 5 anos de idade. Esse valor influencia na quantidade de partícula empregada na formação do painel, pois está diretamente relacionado ao seu peso.

\section{Massa Específica Aparente (MEA) dos Painéis}

A massa específica aparente (MEA) média dos painéis por tratamento é apresentada na Tabela 2. A ANOVA indicou diferença significativa entre as MEA's. A pequena diferença ocorrida na MEA dos painéis pode estar relacionada à adição do aditivo mineral na composição destes. Por causa desse fato, foi realizada a análise de covariância (ANCOVA), a fim de se eliminar o efeito da MEA sobre as propriedades avaliadas. Para tanto, foi realizado o ajuste das MEA's, obtendo-se a média ajustada $\left(\mathrm{MEA}_{\mathrm{A}}\right)$ pela ANCOVA.

TABELA 2: Massa específica aparente média dos painéis sem ajuste (MEA) e com ajuste $\left(\mathrm{MEA}_{\mathrm{A}}\right)$ por tratamento.

TABLE 2: Average density of the wood-cement bonded particleboards ajusted and not ajusted for each treatment.

\begin{tabular}{c|c|c|c}
\hline \multirow{2}{*}{ Tratamento } & \multicolumn{3}{|c}{ Massa específica aparente dos painéis } \\
\cline { 2 - 4 } & $\begin{array}{r}\text { MEA }(12 \%) \\
\left(\mathrm{g} / \mathrm{cm}^{3}\right)\end{array}$ & $\begin{array}{c}\mathrm{CV} \\
(\%)\end{array}$ & $\begin{array}{r}\mathrm{MEA}_{\mathrm{A}} \\
\left(\mathrm{g} / \mathrm{cm}^{3}\right)\end{array}$ \\
\hline $\mathrm{T}_{1}$ & $1,339 \mathrm{a}$ & 0,60 & \\
$\mathrm{~T}_{2}$ & $1,385 \mathrm{~b}$ & 1,58 & \\
$\mathrm{~T}_{3}$ & $1,351 \mathrm{ab}$ & 0,85 & 1,350 \\
$\mathrm{~T}_{4}$ & $1,345 \mathrm{a}$ & 0,36 & \\
$\mathrm{~T}_{5}$ & $1,344 \mathrm{a}$ & 0,76 & \\
$\mathrm{~T}_{6}$ & $1,350 \mathrm{ab}$ & 0,66 & \\
$\mathrm{~T}_{7}$ & $1,345 \mathrm{a}$ & 0,57 & \\
$\mathrm{~T}_{8}$ & $1,366 \mathrm{ab}$ & 0,99 & \\
$\mathrm{~T}_{9}$ & $1,387 \mathrm{~b}$ & 1,70 & \\
\hline
\end{tabular}

Em que: Médias seguidas por letras distintas dentro da mesma coluna mostram diferença estatística ao nível de 95\% de probabilidade de acordo com o teste de Tukey. $\mathrm{CV}=$ Coeficiente de variação.

\section{Propriedades Mecânicas}

\section{Ligação interna (LI)}

Os valores médios ajustados pela ANCOVA para LI são apresentados na Tabela 3. Os resultados da ANCOVA indicaram que os fatores isoladamente influenciaram significativamente a propriedade, assim como a sua interação. 
TABELA 3: Valores médios para a propriedade de Ligação interna (LI) dos painéis.

TABLE 3: Average values for the Internal Bonding (IB) of the wood-cement bonded particleboards.

\begin{tabular}{|c|c|c|c|c|}
\hline \multicolumn{3}{|c|}{ Fonte de Variação } & \multicolumn{2}{|c|}{ Ligação Interna } \\
\hline \multicolumn{3}{|c|}{ Fatores/Níveis - Interações } & $\begin{array}{l}\text { Resistência } \\
\text { (MPa) }\end{array}$ & $\begin{array}{l}\text { CV } \\
(\%)\end{array}$ \\
\hline \multicolumn{5}{|c|}{ A - Relação Madeira:Casca } \\
\hline \multicolumn{3}{|c|}{$1-100: 0$} & 0,68 A & - \\
\hline \multicolumn{3}{|c|}{$2-95: 5$} & $0,59 \mathrm{~b}$ & - \\
\hline \multicolumn{3}{|c|}{$3-90: 10$} & $0,56 \quad \mathrm{c}$ & - \\
\hline \multicolumn{5}{|c|}{ B - Percentual de Aditivo } \\
\hline \multicolumn{3}{|c|}{$1-0$} & $0,59 a b$ & - \\
\hline \multicolumn{3}{|c|}{$2-20$} & 0,65 a & - \\
\hline \multicolumn{3}{|c|}{$3-30$} & $0,58 \quad b$ & - \\
\hline \multicolumn{5}{|c|}{ A x B - Interação } \\
\hline $\mathrm{T}_{1}$ & $\mathrm{~A}_{1} \times \mathrm{B}_{1}$ & $(100: 0-0)$ & 0,68 a & 7,53 \\
\hline $\mathrm{T}_{2}$ & $\mathrm{~A}_{1} \times \mathrm{B}_{2}$ & $(100: 0-20)$ & 0,74 a & 6,95 \\
\hline $\mathrm{T}_{3}$ & $\mathrm{~A}_{1} \times \mathrm{B}_{3}$ & $(100: 0-30)$ & $0,61 \quad b$ & 8,39 \\
\hline $\mathrm{T}_{4}$ & $\mathrm{~A}_{2} \times \mathrm{B}_{1}$ & $(95: 5-0)$ & $0,56 \quad b$ & 9,22 \\
\hline $\mathrm{T}_{5}$ & $\mathrm{~A}_{2} \times \mathrm{B}_{2}$ & $(95: 5-20)$ & 0,63 a & 8,13 \\
\hline $\mathrm{T}_{6}$ & $\mathrm{~A}_{2} \times \mathrm{B}_{3}$ & $(95: 5-30)$ & $0,59 \mathrm{ab}$ & 8,71 \\
\hline $\mathrm{T}_{7}$ & $\mathrm{~A}_{3} \times \mathrm{B}_{1}$ & $(90: 10-0)$ & $0,54 \mathrm{a}$ & 9,48 \\
\hline $\mathrm{T}_{8}$ & $\mathrm{~A}_{3} \times \mathrm{B}_{2}$ & $(90: 10-20)$ & 0,58 a & 8,81 \\
\hline $\mathrm{T}_{9}$ & $\mathrm{~A}_{3} \times \mathrm{B}_{3}$ & $(90: 10-30)$ & $0,54 \mathrm{a}$ & 9,45 \\
\hline
\end{tabular}

Em que: Letras minúsculas diferentes dentro da mesma seção e coluna denotam médias estatisticamente diferentes a 95\% de probabilidade pelo teste de Tukey; $\mathrm{CV}=$ Coeficiente de variação.

Os valores médios de resistência à LI para o fator A (relação madeira:casca) indicaram diferença significativa entre os três níveis da relação, sendo a relação madeira:casca 100:0, aquela com maior valor médio (0,68 MPa), enquanto a relação madeira:casca 90:10 gerou menor valor médio de LI (0,56 MPa).

Para o fator B (percentagem de aditivo) não houve diferença significativa entre os percentuais de aditivo 0 e $20 \%$, com resistência à LI de 0,59 e $0,65 \mathrm{MPa}$ respectivamente. Não houve também diferença significativa entre 0 e $30 \%$ de aditivo. A diferença estatística foi verificada entre os percentuais de aditivos 20 e $30 \%$.

A interação entre os fatores (A x B) foi significativa, exceto quando se adiciona $10 \%$ de casca na mistura. Para a relação madeira:casca 100:0, o desdobramento da interação mostrou que não houve diferença significativa entre os valores obtidos entre os painéis produzidos sem a presença do aditivo $(0 \%)$ e aqueles produzidos com $20 \%$ de aditivo mineral. Os valores médios obtidos por estes foram de 0,68 e $0,74 \mathrm{MPa}$ respectivamente. Os painéis produzidos com $30 \%$ de aditivo mineral apresentaram valor médio de LI de 0,61 $\mathrm{MPa}$, diferenciando-se estatisticamente dos demais.

Para a relação madeira:casca 95:5, os painéis produzidos sem o aditivo mineral apresentaram diferença estatística de resistência à LI $(0,56 \mathrm{MPa})$ daqueles produzidos com $20 \%$ de aditivo $(0,63 \mathrm{MPa})$, enquanto que a adição de $30 \%$ na composição dos painéis não gerou diferença significativa das demais composições, apresentando valor médio de $0,59 \mathrm{MPa}$.

Para a relação 90:0 não foi observada diferença estatística de resistência à LI para as três porcentagens de aditivo mineral aplicadas na composição dos painéis.

Em estudo realizado por Latorraca (2000), no qual utilizou-se $10 \%$ de microssílica em painéis cimento-madeira de Eucalyptus. urophylla, o maior valor obtido de LI foi de 0,30 MPa.

Nota-se, de maneira geral, que a adição de $20 \%$ de microssílica tende a elevar os valores de LI nas três relações madeira:casca, embora em sua maioria, não tenham apresentado resultados estatisticamente diferentes. 


\section{Módulo de elasticidade (MOE)}

Os valores médios de MOE são apresentados na Tabela 4. Analisando-se a relação madeira:casca para a propriedade $\mathrm{MOE}$, verifica-se que não houve diferença significativa quando os painéis foram compostos pela adição de 5 e de 10\% de casca, apresentando valores de MOE de 2111,9 e 1882,09 MPa respectivamente. Entretanto, a relação madeira:casca 100:0 diferenciou-se das outras duas, apresentando valor de MOE de 2885,90 MPa.

Para o fator percentagem de aditivo separadamente, nota-se que não houve diferença significativa de MOE entre aqueles painéis produzidos sem a presença do aditivo mineral $(2256,94 \mathrm{MPa})$ e aqueles com adição de 20 e $30 \%$ de aditivo. A diferença foi observada entre essas duas últimas porcentagens cujos valores médios foram de 2469,31 e 2153,65 MPa respectivamente.

A interação dos fatores indicou igualdade de MOE para os painéis produzidos sem a adição do aditivo mineral e aqueles produzidos com $20 \%$ de aditivo dentro da relação madeira:casca 100:00 com 3224,2 e 3053,78 MPa respectivamente. Ainda para os painéis sem casca, a adição de $30 \%$ de microssílica reduziu significativamente a rigidez dos painéis, apresentando valor médio de 2379,83 MPa.

Dentro da relação madeira:casca 95:5, observou-se diferença estatística entre os tratamentos compostos por 0 e $20 \%$ de aditivo mineral, com valores de MOE de 1989,99 e 2257,14 MPa respectivamente. Os painéis produzidos com $30 \%$ de aditivo não apresentaram diferença estatística dos demais, com valor médio de 2088,77 MPa.

Para a relação madeira:casca 90:10, os painéis produzidos sem o aditivo mineral (MOE de 1556,73 $\mathrm{MPa}$ ) foram estatisticamente diferentes daqueles produzidos com 20 e $30 \%$ de aditivo, apresentando valor médio de MOE de 2096,91 e 1992,54 MPa respectivamente.

Nota-se que com o aumento da casca na composição dos painéis, a adição de 20 e $30 \%$ eleva a rigidez dos painéis. Gerotto et al. (2000) observaram, em trabalho realizado com aditivos minerais em composição com o cimento Portland, que a microssílica tem sido amplamente utilizada em razão do seu tamanho micrométrico e forma arredondada. Essas qualidades proporcionam, na matriz do cimento, melhora de suas resistências mecânicas, especialmente a rigidez.

\section{Módulo de ruptura (MOR)}

Os valores médios de MOR, apresentados na Tabela 4, mostram que, separadamente, os fatores geraram diferença significativa entre os níveis avaliados.

Para o fator relação madeira:casca, os três níveis, diferiram estatisticamente entre si, sendo que os painéis sem adição de casca apresentaram o maior valor médio (21,01 MPa), enquanto que níveis maiores (90:5 e 90:10) de inclusão de casca contribuíram para diminuir a resistência dos Painéis.

O fator percentagem de aditivo não mostrou diferença significativa entre os painéis produzidos com 20 e $30 \%$ de aditivo. Esses painéis geraram maiores valores médios em relação àqueles produzidos sem a adição de microssílica.

Houve efeito significativo da interação entre os fatores para o MOR. Notou-se que, dentro da relação madeira:casca 100:00, não há uma tendência clara quanto ao efeito do aditivo. A adição de $30 \%$ de microsílica reduziu efetivamente o MOR, entretanto a adição de $20 \%$ não demonstrou alteração significativa na resistência dos painéis.

Quando se adicionou 5 ou $10 \%$ de casca na mistura a adição de microssílica influenciou significativamente a resistência dos painéis. Não foi observada diferença significativa do MOR entre adições de 20 e $30 \%$ de microssílica. Portanto, a combinação de casca, até o nível estudado (10\%), mais o aditivo mineral proporcionou painéis com resistência superior àquelas sem adição de casca (100:0).

\section{Propriedades Físicas}

\section{Inchamento em espessura em 2 horas (IE 2 h)}

Os valores médios de IE $2 \mathrm{~h}$ são apresentados na Tabela 5. Para o fator madeira:casca, a composição 100:0 apresentou menor variação dimensional $(0,64 \%)$, diferenciando-se estatisticamente das outras duas composições. Para o fator aditivo, essa diferença estatística foi observada quando se adicionaram 20 e $30 \%$ 
de aditivo, proporcionando menores variações dimensionais $(0,67$ e $0,73 \%)$, em relação à média dos painéis sem a presença do aditivo. Ou seja, a presença do aditivo, independente da percentagem e relação madeira:casca, causou efeito na variação dimensional dos painéis.

Avaliando-se a interação dos fatores, observou-se que, para a relação madeira:casca 100:0, os três níveis de aditivos não geraram diferença significativa entre si.

TABELA 4: Valores médios de Módulo de Elasticidade (MOE) e Módulo de Ruptura (MOR) dos painéis de cimento-madeira.

TABLE 4: Average values of Modulus of Elasticity (MOE) and Modulus of Rupture (MOR) of the wood-cement bonded particleboards.

\begin{tabular}{|c|c|c|c|c|c|c|c|}
\hline \multicolumn{3}{|c|}{ Fonte de Variação } & \multicolumn{5}{|c|}{ Flexão Estática } \\
\hline \multicolumn{3}{|c|}{ Fatores/Níveis - Interações } & $\begin{array}{l}\mathrm{MOE} \\
\text { (Mpa) }\end{array}$ & & $\begin{array}{l}\mathrm{CV} \\
(\%)\end{array}$ & $\begin{array}{l}\text { MOR } \\
\text { (Mpa) }\end{array}$ & $\begin{array}{l}\text { CV } \\
(\%)\end{array}$ \\
\hline \multicolumn{8}{|c|}{ A - Relação Madeira:Casca } \\
\hline \multicolumn{3}{|c|}{$1-100: 0$} & 2885,90 & $\mathrm{a}$ & - & 21,01 a & - \\
\hline \multicolumn{3}{|c|}{$2-95: 5$} & 2111,90 & $\mathrm{~b}$ & - & $19,39 \quad b$ & - \\
\hline \multicolumn{3}{|c|}{$3-90: 10$} & 1882,09 & $\mathrm{~b}$ & - & 14,66 & - \\
\hline \multicolumn{8}{|c|}{ B - Percentual de Aditivo } \\
\hline \multicolumn{3}{|c|}{$1-0 \%$} & 2256,94 & $a b$ & - & $16,10 \quad b$ & - \\
\hline \multicolumn{3}{|c|}{$2-20 \%$} & 2469,31 & $\mathrm{a}$ & - & 19,79 a & - \\
\hline \multicolumn{3}{|c|}{$3-30 \%$} & 2153,65 & $\mathrm{~b}$ & - & 19,18 a & - \\
\hline \multicolumn{8}{|c|}{ A x B - Interação } \\
\hline $\mathrm{T}_{1}$ & $\mathrm{~A}_{1} \times \mathrm{B}_{1}$ & $(100: 0-0)$ & 3224,20 & $\mathrm{a}$ & 8,56 & $20,75 \mathrm{ab}$ & 11,79 \\
\hline $\mathrm{T}_{2}$ & $\mathrm{~A}_{1} \times \mathrm{B}_{2}$ & $(100: 0-20)$ & 3053,79 & $\mathrm{a}$ & 9,04 & 21,83 a & 11,16 \\
\hline $\mathrm{T}_{3}$ & $\mathrm{~A}_{1} \times \mathrm{B}_{3}$ & $(100: 0-30)$ & 2379,83 & $\mathrm{~b}$ & 11,60 & $20,49 \quad b$ & 11,88 \\
\hline $\mathrm{T}_{4}$ & $\mathrm{~A}_{2} \times \mathrm{B}_{1}$ & $(95: 5-0)$ & 1989,99 & $\mathrm{~b}$ & 13,88 & $17,02 \quad b$ & 14,31 \\
\hline $\mathrm{T}_{5}$ & $\mathrm{~A}_{2} \times \mathrm{B}_{2}$ & $(95: 5-20)$ & 2257,14 & $\mathrm{a}$ & 12,23 & 20,99 a & 11,60 \\
\hline $\mathrm{T}_{6}$ & $\mathrm{~A}_{2} \times \mathrm{B}_{3}$ & $(95: 5-30)$ & 2088,77 & $a b$ & 13,20 & 20,16 a & 12,08 \\
\hline $\mathrm{T}_{7}$ & $A_{3} \times B_{1}$ & $(90: 10-0)$ & 1556,73 & $\mathrm{~b}$ & 17,73 & $10,53 \quad b$ & 23,12 \\
\hline $\mathrm{T}_{8}$ & $A_{3} \times B_{2}$ & $(90: 10-20)$ & 2096,91 & $\mathrm{a}$ & 13,17 & $16,57 \mathrm{a}$ & 14,69 \\
\hline $\mathrm{T}_{9}$ & $A_{3} \times B_{3}$ & $(90: 10-30)$ & 1992,54 & $\mathrm{a}$ & 13,86 & 16,89 a & 14,41 \\
\hline
\end{tabular}

Em que: Letras minúsculas diferentes dentro da mesma seção e coluna denotam médias estatisticamente diferentes a 95\% de probabilidade pelo teste de Tukey; $\mathrm{CV}=$ Coeficiente de variação.

Para as relações 95:5 e 90:10, verificou-se diferença estatística de IE 2 h entre os painéis produzidos sem o aditivo e as demais porcentagens, em que a presença do aditivo mineral reduziu significativamente a sua variação dimensional.

Pardo et al. (2001) afirmam que as partículas finas de microssílica levariam a uma melhor adesão, sobretudo na interface matriz-agregado, e também à diminuição da permeabilidade, o que pode explicar a melhoria da estabilidade dimensional dos painéis com adição de microssílica.

Inchamento em espessura em 24 horas (IE 24 h)

Para os valores de IE 24 h, nota-se que a adição de casca à mistura promoveu aumento significativo do inchamento em espessura dos painéis, assim como observado em IE $2 \mathrm{~h}$. Para o fator percentagem de aditivo, não há uma tendência clara.

A interação entre os fatores indica que, para painéis sem adição de casca 100:0, a adição de microssílica aumenta a estabilidade dimensional destas.

Para a relação 95:5, a variação dimensional média dos painéis produzidos sem a presença do aditivo mineral $(1,51 \%)$ não diferenciou daqueles produzidos com $20 \%$ de aditivo $(1,48 \%)$. Já em relação àqueles produzidos com $30 \%$ de aditivo $(1,38 \%)$ houve diferença estatística.

Para a relação 90:10 não foi observada diferença estatística entre 0 e 30\% de aditivo, ou seja a presença do aditivo mineral não gerou efeito na variação dimensional em espessura após $24 \mathrm{~h}$ de imersão em 
água.

A adição de $20 \%$ de microssílica gerou diferença significativa dos demais e ainda o menor valor médio de variação dimensional $(1,37 \%)$. Esse resultado provavelmente está diretamente ligado à proteção oferecida pela matriz de cimento que envolve a partícula, associada ao efeito da redução de vazios provocado pela adição desse tipo de aditivo.

TABELA 5: Valores médios para a propriedade de inchamento em espessura (IE) em 2 e $24 \mathrm{~h}$ (IE 2 e $24 \mathrm{~h}$ ).

TABLE 5: Average values of thickness swelling (IE) of the wood-cement bonded particleboards after $2 \mathrm{~h}$ and $24 \mathrm{~h}$ of water immersion.

\begin{tabular}{|c|c|c|c|c|c|c|}
\hline \multicolumn{3}{|c|}{ Fonte de Variação } & \multicolumn{4}{|c|}{ Inchamento em Espessura } \\
\hline \multirow{2}{*}{\multicolumn{3}{|c|}{ Fatores/Níveis - Interações }} & IE $2 \mathrm{~h}$ & $\mathrm{CV}$ & IE $24 \mathrm{~h}$ & $\mathrm{CV}$ \\
\hline & & & \multicolumn{4}{|c|}{$(\%)$} \\
\hline \multicolumn{7}{|c|}{ A - Relação Madeira:Casca } \\
\hline \multicolumn{3}{|c|}{$1-100: 0$} & 0,64 a & - & 1,32 a & - \\
\hline \multicolumn{3}{|c|}{$2-95: 5$} & $0,88 \quad b$ & - & $1,46 \quad b$ & - \\
\hline \multicolumn{3}{|c|}{$3-90: 10$} & 0,83 & - & $1,50 \quad \mathrm{~b}$ & - \\
\hline \multicolumn{7}{|c|}{ B - Percentual de Aditivo } \\
\hline \multicolumn{3}{|c|}{$1-0$} & $0,95 \quad b$ & - & $1,51 \quad b$ & - \\
\hline \multicolumn{3}{|c|}{$2-20$} & $0,67 \mathrm{a}$ & - & 1,35 a & - \\
\hline \multicolumn{3}{|c|}{$3-30$} & 0,73 a & - & $1,41 \mathrm{ab}$ & - \\
\hline \multicolumn{7}{|c|}{ A x B - Interação } \\
\hline $\mathrm{T}_{1}$ & $\mathrm{~A}_{1} \times \mathrm{B}_{1}$ & $(100: 0-0)$ & 0,68 a & 12,88 & $1,46 \quad b$ & 7,66 \\
\hline $\mathrm{T}_{2}$ & $\mathrm{~A}_{1} \times \mathrm{B}_{2}$ & $(100: 0-20)$ & 0,56 a & 15,64 & $1,20 \mathrm{a}$ & 9,32 \\
\hline $\mathrm{T}_{3}$ & $A_{1} \times B_{3}$ & $(100: 0-30)$ & 0,69 a & 12,69 & $1,29 \mathrm{a}$ & 8,67 \\
\hline $\mathrm{T}_{4}$ & $\mathrm{~A}_{2} \times \mathrm{B}_{1}$ & $(95: 5-0)$ & $1,12 \quad b$ & 7,82 & $1,51 \quad b$ & 7,41 \\
\hline $\mathrm{T}_{5}$ & $\mathrm{~A}_{2} \times \mathrm{B}_{2}$ & $(95: 5-20)$ & 0,76 a & 11,52 & $1,48 a b$ & 11,53 \\
\hline $\mathrm{T}_{6}$ & $\mathrm{~A}_{2} \times \mathrm{B}_{3}$ & $(95: 5-30)$ & $0,77 \mathrm{a}$ & 11,38 & $1,38 \mathrm{a}$ & 11,38 \\
\hline $\mathrm{T}_{7}$ & $\mathrm{~A}_{3} \times \mathrm{B}_{1}$ & $(90: 10-0)$ & $1,05 \quad b$ & 8,34 & $1,56 \quad b$ & 8,34 \\
\hline $\mathrm{T}_{8}$ & $A_{3} \times B_{2}$ & $(90: 10-20)$ & $0,70 \mathrm{a}$ & 12,51 & $1,37 \mathrm{a}$ & 12,51 \\
\hline $\mathrm{T}_{9}$ & $\mathrm{~A}_{3} \times \mathrm{B}_{3}$ & $(90: 10-30)$ & $0,74 \mathrm{a}$ & 11,84 & $1,57 \quad b$ & 11,84 \\
\hline
\end{tabular}

Em que: Letras minúsculas diferentes dentro da mesma seção e coluna denotam médias estatisticamente diferentes a 95\% de probabilidade pelo teste de Tukey; $\mathrm{CV}=$ Coeficiente de variação.

Absorção de água em 2 horas (AA 2 h)

Os valores médios de AA $2 \mathrm{~h}$ são apresentados na Tabela 6. Para a relação madeira:casca, isoladamente, a inclusão em níveis crescentes de casca à mistura provocou aumentos proporcionais na absorção de água dos painéis. O maior valor médio de absorção foi observado para a relação 90:10 (6,51 \%).

Para o fator aditivo, isoladamente, a adição de microssílica à mistura diminui significativamente a absorção de água, sendo que a adição de $20 \%$ de aditivo promoveu a menor absorção de água $(5,18 \%)$ diferindo-se estatisticamente dos demais.

A interação dos fatores para AA 2 h mostrou que, para a relação madeira:casca 100:0, o valor médio dos painéis produzidos sem a presença de aditivo não gerou diferença estatística daqueles produzidos com $30 \%$, enquanto que aqueles produzidos com $20 \%$ do aditivo apresentaram diferença significativa dos demais promovendo menor valor médio de absorção $(4,14 \%)$.

Para a relação 95:5, os três níveis de aditivo mineral diferiram estatisticamente. O maior valor médio de absorção foi para os painéis produzidos sem o aditivo mineral $(6,23 \%)$ e o menor para aqueles em que foram adicionados $20 \%$ de aditivo $(5,18 \%)$.

Na relação 90:10, os painéis produzidos sem aditivo geraram diferença estatística e maiores valores médios de absorção $(7,10 \%)$ em relação àqueles produzidos com $20(6,20)$ e 30\% $(6,22 \%)$ de aditivo.

Em valor numérico, a adição de $20 \%$ do aditivo gerou menor absorção de água. Gerotto et al. (2000) citam que, com a adição de microssílica, a porosidade da matriz do cimento é substancialmente reduzida. A 
forma e tamanho da microssílica certamente favorecem a impermeabilidade do compósito.

TABELA 6: Valores médios para a propriedade Absorção de água (AA) 2 e 24 h (AA 2 e 24 h).

TABLE 6: Average values of water absorption (AA) of the wood-cement bonded particleboards after $2 \mathrm{~h}$ and $24 \mathrm{~h}$ of water immersion.

\begin{tabular}{c|c|c|c|c}
\hline Fonte de Variação & \multicolumn{4}{|c}{ Absorção de Água } \\
\hline \multirow{2}{*}{ Fatores/Níveis - Interações } & AA 2 h & CV & AA 24 h & CV \\
\cline { 2 - 4 } & \multicolumn{4}{|c}{$(\%)$} \\
\hline
\end{tabular}

A - Relação Madeira:Casca

$1-100: 0$

$2-95: 5$

$3-90: 10$

\section{4,98 a}

5,82 b

B - Percentual de Aditivo

$1-0$

$2-20$

$6,51 \quad c$

9,39 a

$10,63 \quad b$

- $9,67 \mathrm{a}$

$3-30$

6,31

c

A x B - Interação

$\mathrm{T}_{1} \quad \mathrm{~A}_{1} \times \mathrm{B}_{1}$

$\mathrm{T}_{2} \quad \mathrm{~A}_{1} \times \mathrm{B}_{2}$

$\mathrm{T}_{3} \quad \mathrm{~A}_{1} \times \mathrm{B}_{3}$

$\mathrm{T}_{4} \quad \mathrm{~A}_{2} \times \mathrm{B}_{1}$

5,18 a

5,82 b

- $\quad 10,46 \quad b$

5,82 b

8,83 a

$10,41 \quad b$

$\mathrm{T}_{5} \quad \mathrm{~A}_{2} \times \mathrm{B}$

$\mathrm{T}_{6} \quad \mathrm{~A}_{2} \times \mathrm{B}_{3}$

$(100: 0-0)$

$5,61 \quad b$

13,09

8,72 a

4,14 a

17,74

8,79 a

$(100: 0-30)$

$5,20 \quad \mathrm{~b}$

14,13

$10,67 \quad b$

$(95: 5-0)$

6,23 c

11,79

$10,71 \quad b$

14,18

9,92 a

$(95: 5-30)$

5,18 a

12,14

11,28 c

6,05 b

10,35

$11,96 \quad \mathrm{c}$

6,20 a

11,85

7,76 a

11,81

$9,29 \mathrm{~b}$

$-$

$\mathrm{T}_{8} \quad \mathrm{~A}_{3} \times \mathrm{B}_{2}$

(90:10 - 30)

6,22 a

Em que: Letras minúsculas diferentes dentro da mesma seção e coluna den
$95 \%$ de probabilidade pelo teste de Tukey; $\mathrm{CV}=$ Coeficiente de variação.

Absorção de água em 24 horas (AA 24 h)

Para a variável AA $24 \mathrm{~h}$, o fator madeira:casca, isoladamente, não proporcionou diferença entre as relações 100:0 e 90:10, com valores de 9,39 e 9,67\% respectivamente, e a maior absorção foi verificada para a relação 95:05 com 10,63\%. Para o fator percentual de aditivo, isoladamente, a adição de $20 \%$ de microssílica gerou menor valor médio de AA 24 h (8,83\%), diferenciando-se das demais composições.

A interação entre os fatores indicou igualdade estatística entre os valores médios de absorção daqueles painéis produzidos sem a presença de aditivo (8,72\%) e aqueles produzidos com $20 \%(8,79 \%)$. A adição de $30 \%$ de aditivo gerou maior valor médio de absorção (10,67\%), gerando diferença estatística dos demais.

Para a relação 95:5, houve diferença significativa entre os três níveis de aditivo. O menor valor médio de AA $24 \mathrm{~h}$ foi para os painéis produzidos com $20 \%$ de aditivo $(9,92 \%)$, enquanto que a adição de $30 \%$ de aditivo promoveu a maior absorção $(11,28 \%)$.

Para a relação 90:10 também foi observada diferença estatística entre os três níveis de aditivo. A adição de $20 \%$ gerou menor valor médio $(7,76 \%)$, e a ausência do aditivo promoveu a maior absorção $(11,96 \%)$.

\section{CONCLUSÕES}

A propriedade de LI não sofre efeito significativo do aditivo mineral quando os painéis apresentam $10 \%$ de casca em sua composição. A adição de $20 \%$ de aditivo faz-se necessária apenas quando o painel apresenta em sua composição $5 \%$ de casca.

Para a variável MOE, a adição de $30 \%$ de microssílica tende a reduzir a rigidez do painel quando este é composto por $100 \%$ de madeira, enquanto que a adição de $20 \%$ do aditivo produz aumento da rigidez quando o painel apresenta casca em sua composição. 
O efeito da adição de 20 e $30 \%$ de microssílica em painéis compostos pela relação madeira:casca 95:5 e 90:10 resulta em aumento de MOR.

A adição de 20 e $30 \%$ de aditivo mineral em painéis produzidos com as relações madeira:casca 95:5 e 90:10 reduz a variação dimensional em espessura após $2 \mathrm{~h}$ de imersão em água, enquanto que para a relação 100:0 aditivo não promove efeito significativo.

A adição de $20 \%$ de microssílica proporciona menor variação em espessura após 24 h de imersão em água, para painéis produzidos pela relação madeira:casca 100:0.

A adição de $20 \%$ de microssílica tende a reduzir a absorção de água após 2 e 24 h de imersão em água dentro de todas as relações madeira:casca.

Avaliando-se o conjunto das propriedades estudadas, a inclusão da casca pode ser viabilizada com a adição de $20 \%$ de microssílica na composição dos painéis, melhorarando as propriedades físicas e mecânicas dos painéis.

\section{REFERÊNCIAS BIBLIOGRÁFICAS}

AMERICAN SOCIETY FOR TESTING AND MATERIAL. Standard methods of evaluating the properties of woodbase fiber and particle panel materials. In: Annual Book of ASTM Standard. Philadelphia, 1999. (ASTM D 1037)

CHAPOLA, G.B.J. Effect of curing heat, alkali and cement set in cement particleboard on the visco-elastic properties of chip mattresses. Wood Science and Technology, v. 23, p. 131-138, 1989.

DEUTSCHES INSTITUT FÜR NORMUNG. Testing of wood chipboards, bending test, determination of bending strength. DIN 52362, p. 39-40, 1982

GEROTTO, M.V.; CABO, S.S.; INNOCENTINI, M.D.M.; PANDOLFELLI, V.C. Utilização de caulim e meta-caulim em substituição à microssílica em concretos refratários aluminosos auto-escoantes. Cerâmica, v. 46, n. 300, p. 200209, 2000.

LANGE, H.; SIMATUPANG, M.H.; NEUNAUER, A. Influence of latent hydraulic binders on the properties of woodcement composite. In: INORGANIC BONDED WOOD AND FIBER COMPOSITE MATERIALS. Session II: Raw material considerations. Proceedings... USA. V. 1, 1989, p. 48-52

LATORRACA, J.V.F. Eucalyptus spp. na produção de painéis de cimento-madeira. 2000. 191p. Tese (Doutorado em Ciências Florestais) - Universidade Federal do Paraná, Curitiba, 2000.

PARDO, A.R.F.; INNOCENTINI, M.D.M.; MENEGAZZO, B.; PANDOLFELLI, V.C. Permeabilidade de concretos refratários avançados. Cerâmica, v. 47, n. 302, p. 82-87, 2001.

SOUZA, M.R. Durability of cement-bonded particleboard made conventionally and carbon dioxide injection. 1994. 123p. Tese (Doctor of Philosophy) - University of Idaho, Idaho, 1994. 